\title{
Slack and Net Technical Efficiency Measurement: A Bootstrap Approach
}

\author{
J. Richmond \\ Department of Economics \\ University of Essex \\ Colchester CO4 3SQ \\ U. K.
}

Version 1.0: September 2001

JEL Classification Code: D24

Keywords: Data Envelopment Analysis; Bootstrap; Efficiency measurement. 


\section{INTRODUCTION.}

The analysis of production frontiers and the related problem of efficiency measurement has generated a substantial flow of research in recent years. The historical roots of a rigorous approach to efficiency measurement can be traced to the work of Koopmans (1951), Debreu (1951) and Farrell (1957). This work gave a foundation for the important techniques of Data Envelopment Analysis (henceforth DEA); the term DEA covers a variety of related procedures for efficiency measurement, almost all of which rely on linear programming as the primary computational tool. Excellent recent accounts of the techniques include Färe et al. (1994) and Charnes et al. (1997).

Until recently almost all of the empirical research in this area comprised reports of various efficiency measures but without any accompanying statistical assessment of the reliability of the measures. The reason for this is that the statistical properties of parameter estimates obtained as the solution to linear programming problems are poorly understood, so that properties of the probability distributions of these estimators are for the most part unknown. This situation has begun to change within the last decade, following seminal work by Banker (1993). Some of the recent developments are surveyed in Simar and Wilson (1999). Although more is now known than before, the problems associated with obtaining exact finite sample or even asymptotic distribution theoretical results in this area remain quite intractable. Consequently, in the current state of knowledge, it is necessary to rely to a considerable extent on simulation techniques such as bootstrap methods. Application of bootstrap methods to DEA requires some care, as has been pointed out by Simar and Wilson (1998, 1999, 2000).

The aim of this article is to extend the applicability of the bootstrap approach to a non-radial efficiency measure. The standard DEA input-oriented measure of technical efficiency is a gross measure that does not take into account slack in resource usage. Section two of this article describes a modified net technical efficiency measure which can be viewed as a decomposition of efficiency as the product of the standard (gross) technical 
efficiency measure and a slack efficiency index. In section two of the article, these measures are embodied in a statistical model of production that is suitable for bootstrap simulation. Section three of the article describes in detail how to conduct bootstrap simulation of the model and section four summarizes the results of an empirical application. Section five summarises and concludes the article.

\section{A STOCHASTIC DATA ENVELOPMENT ANALYSIS MODEL.}

The approach adopted throughout the remainder of the paper is based on an inputoriented DEA model. For simplicity in describing the model it is assumed that only one output is produced, although this assumption is easy to relax. An initial description of the model can then be given in terms of a production function which is interpreted, as usual in studies of efficiency, as a frontier function. Thus, only fully efficient use of inputs leads to output corresponding to a point on the production frontier. A distinction is therefore made between the observed input vector and the effective input vector. The effective input vector differs from the observed input vector because of, on the one hand slack inputs, and on the other hand technically inefficient use of the inputs employed. The distinction between slack and technical inefficiency can best be grasped by a simple example. Suppose three firms are being compared and assume that the first of these can produce one unit of output with inputs of one machine operated by one person. For simplicity assume also that the firms being compared all pay the same prices for factor inputs. If another firm operating the same machine with the same labour input produces less than one unit of output, then this second firm is technically inefficient, relative to the first firm. A third firm which does manage to produce one unit of output, but employs two people to operate the machine, has slack in the use of labour. If the standard inputoriented (gross) technical efficiency measure is applied to the situation just described (see (9) below), the technical efficiency index of the first and third firm will be reported as $100 \%$ and if no reference is made to the phenomenon of slack, these two firms would be regarded as fully efficient. Of course, slack and technical inefficiency are not mutually 
exclusive. Typically, both phenomena will be observed together.

Assume that we have observations of inputs and outputs of $n$ firms. Let $y_{k} \in R$ denote observed output of firm $k, k=1, \ldots, n$ and let $x_{k}=\left(x_{1 k}, \ldots, x_{m k}\right)^{\prime} \in R^{m}$ denote the firm's observed input vector. Denote by $\hat{x}_{k} \in R^{m}$ the effective input vector of the firm. The firm's technology is given by the production function

$$
y_{k}=f\left(\hat{x}_{k}\right)
$$

where the effective input vector and the actual input vector are related by

$$
\hat{x}_{k}=\theta_{k}\left(x_{k}-h_{k}\right) .
$$

In $(2), h_{k}=\left(h_{1 k}, \ldots, h_{m k}\right) \in R^{m}$ denotes a vector of slacks in input use and $\theta_{k} \in R$ represents the degree of technical efficiency in the use of inputs. It is assumed that $0<\theta_{k} \leq 1, h_{k} \geq 0$ and that $x_{k}-h_{k}>0$. Let

$$
h_{k}=\Gamma_{k} x_{k}
$$

where

$$
\Gamma_{k}=\operatorname{diagonal}\left(\gamma_{1 k}, \ldots, \gamma_{m k}\right)
$$

is a diagonal matrix with elements $\gamma_{i k}, 0 \leq \gamma_{i k}<1$, and define

$$
s_{k}=\theta_{k} h_{k}
$$

If the $\theta_{k}$ and $\gamma_{i k}$ are treated as firm specific random variables, we can interpret this model in the following way. Firm $k$ uses inputs given by $x_{k}$. A random proportion $\gamma_{i k}$ of the $i$ th input used is slack; the net of slack input vector used by the firm is then $x_{k}-h_{k}$. This net of slack input vector is used in a more or less technically efficient manner by the firm, depending on the random value of the technical efficiency parameter $\theta_{k}$, so that the effective input vector of the firm is $\hat{x}_{k}$. Allowing for the effect of technical inefficiency, the effective vector of slack in the inputs is given by $s_{k}$. The effective input vector yields an output $y_{k}$ determined by the production function $f$, assumed to be common to all the firms in the sample. 
Within this framework, the variable $\theta_{k}$ is a direct radial measure of the technical efficiency of the $k$ th firm. This measure of technical efficiency does not take slack resources into account and for many purposes it may be desirable to compute indicators of the overall extent to which inputs are slack as well as input specific measures of the degree of slackness, the $\gamma_{i k}$ in the model.

If data on input prices are available then in the context of the model described above, an obvious overall indicator of slack efficiency is given by one minus the proportion of input costs that could be saved by the elimination of the slack. Let $q_{k} \in R^{m}$ denote the vector of input prices facing the $k$ th firm and define an index of slack efficiency by

$$
S E_{k}=1-q_{k} h_{k} / q_{k} x_{k}
$$

(here and elsewhere, for two vectors $w \in R^{n}$ and $z \in R^{n}$, wz denotes the inner product $\left.\sum_{i=1}^{n} w_{i} z_{i}\right)$. Note that $0<S E_{k} \leq 1$. With this definition of slack efficiency in mind, net technical efficiency of the $k$ th firm can be defined as

$$
\begin{aligned}
T E_{k}^{N} & =S E_{k} \theta_{k} \\
& =\frac{q \hat{x}_{k}}{q x_{k}} .
\end{aligned}
$$

Estimates of $\theta_{k}$ for each $k$ can be computed using standard DEA techniques. The DEA procedure uses linear programming methods and concomitantly produces values for slack variables which can be used to construct measures of slack efficiency. Use the notation $Y=\left(y_{1}, \ldots, y_{n}\right)^{\prime}, X=\left(x_{1}, \ldots, x_{n}\right)^{\prime}$ and $Q=\left(q_{1}, \ldots, q_{n}\right)^{\prime}$ to denote the observations for all the firms in the sample on, respectively, outputs, inputs and input prices. Gross technical efficiency of the $k$ th firm in the sample is measured by

$$
\hat{\theta}_{k}=\min _{\theta_{k}, \lambda_{k}}\left\{\theta_{k}: Y^{\prime} \lambda_{k} \geq y_{k}, X^{\prime} \lambda_{k} \leq \theta_{k} x_{k}, \lambda_{k} \geq 0, \theta_{k} \text { free }\right\}
$$

One approach to measuring slack efficiency is described in Lynde and Richmond (2000). The method proceeds in two steps. The first step is to estimate technical efficiency using (9). This estimate is then used in the second step to estimate the maximum cost saving 
from the elimination of slack by solving the following linear program

$$
\max _{\lambda_{k}, s_{k}}\left\{q_{k} s_{k}: Y^{\prime} \lambda_{k} \geq y_{k}, X^{\prime} \lambda_{k}+s_{k}=\hat{\theta}_{k} x_{k}, \lambda_{k} \geq 0, s_{k} \geq 0\right\}
$$

The linear programs in (9) and (10) are linked by the fact that if $\left(\lambda_{k}, s_{k}\right)$ is feasible for (10) then $\left(\lambda_{k}, \hat{\theta}_{k}\right)$ is feasible and hence optimal for (9). Thus if $\left(\hat{\lambda}_{k}, \hat{s}_{k}\right)$ solves (10) then $\left(\hat{\lambda}_{k}, \hat{\theta}_{k}\right)$ solves $(9)$ and

$$
X^{\prime} \hat{\lambda}_{k}+\hat{s}_{k}=\hat{\theta}_{k} x_{k}
$$

Now define $\hat{h}_{k}$ by

$$
\hat{h}_{k}=\hat{s}_{k} / \hat{\theta}_{k}
$$

Slack efficiency and net technical efficiency can now be estimated by ${ }^{1}$

$$
S E_{k}=1-q_{k} \hat{h}_{k} / q_{k} x_{k}
$$

and

$$
T E_{k}^{N}=S E_{k} \hat{\theta}_{k}
$$

Although it is relatively straightforward to compute the estimators described here, only a little is known concerning the statistical properties of estimators that use DEA techniques. It is only recently that some progress has been made. This work includes articles by Banker (1993), Simar (1996), Grosskopf (1996), Gijbels et al. (1999), and Simar and Wilson (1999). In the single output case DEA estimators of technical efficiency have been shown, under suitable assumptions about the underlying stochastic structure, to be maximum likelihood estimators of the production frontier Banker (1993). For the case of multiple outputs Kneip et al. (1998) have proved consistency of the DEA estimator. In the special case of a single output produced by a single input, the asymptotic distribution of the estimator has been obtained Gijbels et al. (1999). However, for the general case of multiple outputs and inputs, the relevant distribution theory remains to be developed.

\footnotetext{
${ }^{1}$ To avoid notational clutter we use $S E$, not $\widehat{S E}$ in(13)to refer to the estimate of slack efficiency; similarly for $T E^{N}$ and the estimate of $\hat{x}$.
} 
For this reason, the currently feasible approach to statistical inference in the context of DEA methods must largely rely on computer intensive methods such as bootstrap techniques.

\section{A BOOTSTRAP PROCEDURE.}

In this section we describe a procedure for conducting a bootstrap analysis of the efficiency measures discussed in section two; the method outlined here is based upon the work by Simar and Wilson (1998), extended to take account of slack efficiency measures. As these authors point out, the naive approach to generating bootstrap samples that simply uses the empirical distribution function (EDF) of the original data (attaching probability $1 / n$ to each observation) is unsatisfactory in the context of DEA, which involves estimation of a frontier, and can lead to inconsistent estimates of relevant confidence sets. A more sophisticated approach is necessary.

In order to conduct the bootstrap analysis, it is necessary first to clarify the assumptions made concerning the underlying stochastic model. The firm specific random variables in the model are

$$
\eta_{k}=\left(\theta_{k}, \gamma_{1 k}, \ldots, \gamma_{m k}\right), \quad k=1, \ldots, n
$$

We shall assume that the elements of $\eta_{k}$ are continuous random variables with joint distribution function $G$, which is the same for all $k$, and that the set of random variables $\left\{\eta_{k}\right\}$ are independent. The support of the distribution function is the set $\left\{\eta_{k}=\right.$ $\left.\left(\theta_{k}, \gamma_{1 k}, \ldots, \gamma_{m k}\right): 0<\theta_{k} \leq 1,0 \leq \gamma_{i k}<1, i=1, \ldots, m\right\}$

From (2) and (3)

$$
\hat{x}_{k}=\theta_{k}\left(I-\Gamma_{k}\right) x_{k}
$$

Inverting this equation we have

$$
x_{k}=\left(I-\Gamma_{k}\right)^{-1} \theta_{k}^{-1} \hat{x}_{k} .
$$


Conditional on $\hat{x}_{k}$ and $y_{k}$, we may regard the data generating process for the $x_{k}$ as that summarized by (17) and the distribution function $G$.

To perform a bootstrap simulation, artificial samples of data are required. To do so, it is first necessary to estimate $\theta_{k}$, the $\gamma_{i k}$ and $\hat{x}_{k}$ for each $k$. The gross technical efficiency estimate $\hat{\theta}_{k}$ is obtained from (9). Estimates of the individual input slack efficiency parameters are given by

$$
\hat{\gamma}_{i k}=\hat{h}_{i k} / x_{i k}
$$

where the $\hat{h}_{i k}$ are obtained from (12). An estimate of the effective input vector $\hat{x}_{k}$ can then be obtained by substituting these estimates in (2). The next step is to use the estimates $\hat{\theta}_{k}$ and $\hat{\gamma}_{i k}$ to generate intermediate pseudo sample estimates $\hat{\theta}_{k}^{++}$and $\hat{\gamma}_{i k}^{++}$; the precise method for doing so will be described shortly. Treating the observations on input prices as fixed, and conditional on the observed outputs $y_{k}$ and the observed (estimated) effective input vectors $\hat{x}_{k}$, we can now generate pseudo sample values

$$
x_{k}^{*}=\left(I-\widehat{\Gamma}_{k}^{++}\right)^{-1}\left(\hat{\theta}_{k}^{++}\right)^{-1} \hat{x}_{k} .
$$

Let $X^{*}=\left(x_{1}^{*}, \ldots, x_{k}^{*}\right)^{\prime}$. The final step in the bootstrap simulation procedure is to use this artificially generated input sample matrix $X^{*}$, together with the observed sample values $Y$ and $Q$, of output and prices respectively, to generate new pseudo sample estimates $\hat{\theta}_{k}^{*}$ and $\hat{\gamma}_{i k}^{*}$ and new net technical efficiency and slack efficiency estimates $T E_{k}^{N *}$ and $S E_{k}^{*}$. This is done by replacing $X$ in (9) and (10) by $X^{*}$ and using (12), (13) and (14). When this process is replicated many times, the distribution of the resulting estimates $\hat{\theta}_{k}^{*}$ and $\hat{\gamma}_{i k}^{*}$ may be used to approximate properties of the the distribution of the DEA estimators of efficiency.

We turn now to the precise method to be used for the generation of the intermediate pseudo sample estimates $\hat{\theta}_{k}^{++}$and $\hat{\gamma}_{i k}^{++}$that are used to produce the values $x_{k}^{*}$. A property of the DEA estimates of $\hat{\theta}_{k}$ and $\hat{\gamma}_{i k}$ is that with probability one, for some $k$ and $i, \hat{\theta}_{k}=1$ and $\hat{\gamma}_{i k}=0$. Since by assumption the random variables $\theta_{k}$ and $\gamma_{i k}$ are continuous, this has the consequence that the empirical distribution function is a poor estimator of the 
true distribution function of these random variables, at least near the upper boundary of the support of $\theta_{k}$ and the lower boundary of the support of $\gamma_{i k}$. As a result, the simple EDF approach may fail to provide consistent bootstrap confidence sets. Note that in practice, values of $\hat{\theta}_{k}$ equal to zero and values of $\hat{\gamma}_{i k}$ equal to unity are not observed. In each case, the difficulty only arises at one of the boundaries of the support. The bootstrap approach can fail in this situation because the problem of efficiency estimation in effect requires the estimation of a boundary. This is analogous, in the univariate case of a random variable uniformly distributed on $[0, \tau]$, to the estimation of $\tau$ (Wilson and Simar (1995)), a situation in which artificial resampling from the EDF is known to fail to provide accurate confidence sets (see Efron and Tibshirani 1993).

An alternative procedure, recommended by Wilson and Simar (1995) as a way to circumvent this problem with DEA estimation procedures, is based on a modification of the smoothed bootstrap, which uses kernel density estimation techniques. The kernel density estimator is modified by the reflection method of Silverman (1986), to take account of the problem with boundary values associated with the DEA estimates.

Let $V_{1}, \ldots, V_{n}$ denote a random sample of a continuous random variable $V$ with unknown continuous density function $f$. Under fairly general conditions a consistent kernel density estimator of $f$ is given by

$$
\hat{f}(v)=\frac{1}{n b} \sum_{i=1}^{n} K\left(\frac{v-v_{i}}{b}\right)
$$

where $K$ is a continuous and symmetric density function with mean zero and variance equal to unity and $b$ is a bandwidth parameter. Corresponding to $\hat{f}$ is a distribution function estimate $\hat{F}$. Instead of using the EDF to produce artificially simulated resamples of $V$, the estimated distribution function $\hat{F}$ is used. In practice, given the observed sample $v_{1}, \ldots, v_{n}$ simulation is achieved by constructing variables

$$
V_{j}^{*}=v_{I_{j}}+b \epsilon_{j}
$$

where the $I_{j}$ are independent and uniformly distributed on the integers $1, \ldots, n$ and the $\epsilon_{j}$ are a random sample from a variable with density $K$ and are independent of the $I_{j}$. 
Define $Z_{j}=b \epsilon_{j}$; the density function of $Z_{j}$ is $h(z)=(1 / b) K(z / b)$ and by the convolution theorem (Grimmett and Stirzaker 1992, p. 113) the density function for $V_{j}^{*}=v_{I_{j}}+Z_{j}$ is

$$
f_{V_{j}^{*}}(v)=\int_{-\infty}^{\infty} f_{V_{I_{j}}}(x) \frac{1}{b} K\left(\frac{v-x}{b}\right) d x
$$

Since

$$
\begin{aligned}
f_{v_{I_{j}}}(x) & =\frac{1}{n} & \text { if } x=v_{j} \\
& =0 & \text { otherwise }
\end{aligned}
$$

then

$$
\begin{aligned}
f_{V_{j}^{*}}(v) & =\frac{1}{n b} \sum_{i=1}^{n} K\left(\frac{v-v_{i}}{b}\right) \\
& =\hat{f}(v)
\end{aligned}
$$

Thus the $V_{j}^{*}$ defined in (21) have a distribution which is precisely that of the kernel density estimate of $f$. Note that in $(21)$, if $b=0$, the $V_{j}^{*}$ would simply correspond to resampling from the EDF. When the support of the random variable $V$ is bounded, simulation of bootstrap samples using (21) is inappropriate when $b>0$ because it can produce pseudo-sample values that violate the boundary condition.

The reflection method is a modification of this approach to take account of the problem of bounded support (Silverman (1986)). Consider first the $\hat{\gamma}_{i k}$, for which the problem arises at the lower boundary of the support (recall that $0 \leq \gamma_{i k}<1$ ), and reflect around zero in the following way. Define

$$
\begin{aligned}
\hat{\gamma}_{i k}^{+} & =\hat{\gamma}_{i I_{k}}+b_{i} \epsilon_{i k} & \text { if } \hat{\gamma}_{i I_{k}}+b_{i} \epsilon_{i k} \geq 0 \\
& =-\left(\hat{\gamma}_{i I_{k}}+b_{i} \epsilon_{i k}\right) & \text { otherwise }
\end{aligned}
$$

where the $I_{k}$ are independent and uniformly distributed on the integers $1, \ldots, n, b_{i}$ is a bandwidth parameter and the $\epsilon_{i k}$ are a random sample from a variable with density $K$ and are independent of the $I_{k}$. Then it can be shown that the density function of the $\hat{\gamma}_{i k}^{+}$generated in this way is a consistent estimator of the density function of the random 
variables $\hat{\gamma}_{i k}$. For some detail of the argument here, see the appendix to this article. Thus the $\hat{\gamma}_{i k}^{+}$can be treated as approximations to random samples from the distribution of the $\hat{\gamma}_{i k}$.

Similarly, in the case of the $\hat{\theta}_{k}$, for which the relevant boundary is at the upper end of the support $\left(0<\theta_{k} \leq 1\right)$, the reflection method now is applied by reflecting around unity. Now define

$$
\begin{aligned}
\hat{\theta}_{k}^{+} & =\hat{\theta}_{I_{k}}+b \epsilon_{k} \quad \text { if } \hat{\theta}_{I_{k}}+b \epsilon_{k} \leq 1 \\
& =2-\left(\hat{\theta}_{I_{k}}+b \epsilon_{k}\right) \quad \text { otherwise }
\end{aligned}
$$

where $b$ is a bandwidth parameter and the $\epsilon_{k}$ are a random sample from a variable with density $K$ and are independent of the $I_{k}$. The density function of the $\hat{\theta}_{k}^{+}$is a consistent estimator of the density of $\hat{\theta}_{k}$ and the $\hat{\theta}_{k}^{+}$are approximations to random samples from the distribution of the $\hat{\theta}_{k}$.

It is standard practice with kernel estimators to rescale so that the resulting bootstrap samples have the same variance as unsmoothed bootstrap samples. Estimators so modified are commonly referred to as shrunk smoothed bootstrap estimators. In the case considered here this is achieved by the transformation

$$
\hat{\theta}_{k}^{++}=(1-a) \overline{\hat{\theta}}+a \hat{\theta}_{k}^{+}
$$

where

$$
\begin{aligned}
a & =\left(1+b^{2} / \hat{\sigma}^{2}\right)^{-1 / 2} \\
\hat{\sigma}^{2} & =\frac{1}{n} \sum_{i=1}^{n}\left(\hat{\theta}_{k}-\overline{\hat{\theta}}\right)^{2} \\
\overline{\hat{\theta}} & =\frac{1}{n} \sum_{k=1}^{n} \hat{\theta}_{k}
\end{aligned}
$$

and by the transformations

$$
\hat{\gamma}_{i k}^{++}=\left(1-a_{i}\right) \overline{\hat{\gamma}}_{i}+a_{i} \hat{\gamma}_{i k}^{+}
$$


where

$$
\begin{aligned}
a_{i} & =\left(1+b_{i}^{2} / \hat{\sigma}_{i}^{2}\right)^{-1 / 2} \\
\hat{\sigma}_{i}^{2} & =\frac{1}{n} \sum_{k=1}^{n}\left(\hat{\gamma}_{i k}-\overline{\hat{\gamma}}_{i}\right)^{2} \\
\overline{\hat{\gamma}}_{i} & =\frac{1}{n} \sum_{k=1}^{n} \hat{\gamma}_{i k} .
\end{aligned}
$$

Finally, the kernel function $K$ and the bandwidth parameters $b$ and $b_{i}, i=1, \ldots, m$ need to be chosen. For $K$, a simple choice is the density function of the standard Normal distribution; this was used in the application described in the next section. There is a trade-off between choosing values of a bandwidth parameter that is too high, leading to oversmoothing with a loss of efficiency in estimation, and choosing too small a value, which can lead to density estimates with a mode at each data point. Following Simar and Wilson (1998), it was decided to adopt an automatic bandwidth selection procedure recommended in Silverman (1986).

To summarize, values of $\hat{\theta}_{k}^{++}$and $\hat{\gamma}_{i k}^{++}$are generated using (31) and (35). These values are then used in (19) to generate values $x_{k}^{*}$ which in turn can be used to generate new bootstrap pseudo sample estimates $\hat{\theta}_{k}^{*}$ and $\hat{\gamma}_{i k}^{*}$ and new net technical efficiency and slack efficiency estimates $T E_{k}^{N *}$ and $S E_{k}^{*}$. This procedure is repeated many times to generate a set of bootstrap replicate values of these parameters, the empirical distribution of which can be used to approximate statistical properties of the efficiency estimators.

\section{AN EMPIRICAL APPLICATION.}

In this section we briefly describe the results obtained from an application of the bootstrap methodology to the well-known data set used in the study by Christensen and Greene (1976) of electricity generation in the USA. A subset comprising 155 firm level observations for the year 1970 was used $^{2}$. The data include observations of output and

\footnotetext{
${ }^{2} \mathrm{~A}$ few observations for which factor shares did not sum to unity were dropped from the original data set. My thanks to W. H. Greene for making the data available.
} 
total cost, as well as prices and quantities for capital, labour and fuel inputs. A detailed description of the data set may be found in Christensen and Greene (1976). Good descriptions of the bootstrap methodology may be found in Davison and Hinkley (1997), Efron and Tibshirani (1993) and Hall (1992).

A set of estimates of the three efficiency measures $\left\{\hat{\theta}_{k}, S E_{k}, T E_{k}^{N}: k=1, \ldots, n\right\}$ was generated from the original sample using equations (9), (10), (12), (13) and (14). The bootstrap methodology described in section three was then applied to obtain a set of $r$ pseudo estimates for each firm $\left\{\hat{\theta}_{k}^{*}(b), S E_{k}^{*}(b), T E_{k}^{N *}(b): k=1, \ldots, n ; b=1, \ldots, r\right\}$. For each value of $k$, the resulting set of bootstrapped estimates was used to approximate properties of the gross and net technical efficiency and the slack efficiency measures $\hat{\theta}_{k}$, $T E_{k}^{N}$ and $S E_{k}$. The bootstrap estimates were used to compute, for these efficiency measures, estimates of the bias and mean squared error as well as bootstrap confidence intervals. In the case of $\hat{\theta}_{k}$, these estimates were constructed as follows (the computations for $T E_{k}^{N}$ and $S E_{k}$ are similar). The bootstrap estimate of bias is

$$
\begin{aligned}
\hat{B}\left(\hat{\theta}_{k}\right) & =\frac{1}{r} \sum_{b=1}^{r} \hat{\theta}_{k}^{*}(b)-\hat{\theta}_{k} \\
& =\overline{\hat{\theta}}_{k}^{*}-\hat{\theta}_{k},
\end{aligned}
$$

giving the bias-corrected estimate

$$
\tilde{\theta}_{k}=\hat{\theta}_{k}-\hat{B}\left(\hat{\theta}_{k}\right)
$$

To approximate the variance of $\hat{\theta}_{k}$, the bootstrap estimate

$$
\tilde{\sigma}_{k}^{2}=\frac{1}{r} \sum_{b=1}^{r}\left(\hat{\theta}_{k}^{*}(b)-\overline{\hat{\theta}}_{k}^{*}\right)^{2}
$$

is used and the mean squared error is then estimated by

$$
M S E_{k}=\tilde{\sigma}_{k}^{2}+\hat{B}\left(\hat{\theta}_{k}\right)^{2}
$$

The bootstrap confidence intervals reported are symmetric bias-corrected intervals, centred at the biased corrected estimates $\tilde{\theta}_{k}$. These are constructed as follows. First transform the bootstrap estimates so as to centre them on $\tilde{\theta}_{k}$. Let $W(b)=\hat{\theta}_{k}^{*}(b)-2 \hat{B}\left(\hat{\theta}_{k}\right)$; 
it is easy to show that the mean value $\bar{W}=\tilde{\theta}_{k}$. Now let $Z(b)=W(b)-\tilde{\theta}_{k}$ and use the empirical distribution of the $Z(b)$ to find $C_{\alpha}$, the $100 \alpha$ percentile of the empirical distribution of the absolute value $|Z(b)|$. The $100 \alpha \%$ bootstrap confidence interval for $\theta_{k}$ is given by

$$
C I_{k}=\left(\tilde{\theta}_{k}-C_{\alpha}, \tilde{\theta}_{k}+C_{\alpha}\right)
$$

In order to conserve space, rather than report a complete set of results for all three efficiency measures for each of the 155 firms in the sample, we report here detailed results for only 15 of the firms analyzed, selected at random, and representing approximately ten percent of the original sample. These results for gross and net technical efficiency and slack efficiency are reported in Tables 1,2 and 3 respectively. In these tables, the final column gives the efficiency ranking in the full sample of the firms selected; thus, for example, the 6th sample observation $(k=6)$ was ranked $123 \mathrm{rd}$ out of the 155 firms in the full sample by the gross technical efficiency measure. Table 4 contains some summary statistics for the full sample of 155 firms. In the results reported in these tables, $r=500$ bootstrap replications were used, and the confidence intervals reported are approximate 95\% confidence intervals. In these tables, the column marked 'BC' denotes the bias-corrected efficiency estimate. Since the estimates of efficiency relate in effect to an estimated production frontier which must lie within the true production frontier, then a priori these estimates are upward biased. In a small number of cases the bootstrap bias estimates indicate downward (negative) bias; in these cases the bias estimate has been truncated at zero. Similarly, in a very few cases the confidence interval for the efficiency estimates is just above unity; these values have been truncated at unity.

The most obvious feature to emerge from the results reported in these tables is that slack efficiency levels, as measured by $S E_{k}$, are typically quite high; most firms have slack efficiency levels of at least 90\%. Consequently the extent to which net and gross technical efficiency levels differ is quite limited, although it is clear that the rank ordering of firms by the slack efficiency measure can differ considerably from that by the gross or net technical 
efficiency measures. Although the efficiency measures are biased, the order of magnitude of the bias is only of the order of $3 \%$ for the technical efficiency measures, and rather less than this for the slack efficiency measure. The technical efficiency measures are typically somewhat lower than the slack efficiency measures, by around $15 \%$ on average over the full sample. This suggests that for most of these firms, the most important source of inefficiency is not slack input usage; instead it appears to be a consequence of technically inefficient use of inputs. ${ }^{3}$ In Table 2, the estimates indicate that five of the 15 firms in the subsample were fully efficient with respect to the slack efficiency measure - even to the extent that the confidence interval width has collapsed to zero. The subsample results for the slack efficiency measure are mirrored in the full sample for which approximately one third of the sample, 56 firms, were fully efficient. Although it can be seen from Tables 1 and 3 that none of the firms in the subsample were fully technically efficient, by either the gross or net measures, in the full sample 11 firms were fully technically efficient by both the gross and net measures.

What does emerge strongly from the bootstrap simulations is the need to exercise some caution in the interpretation of DEA results that are not supplemented by some assessment of the statistical reliability of the efficiency measures. While the bias in these measures appears to be quite small, it is clear from the tables that the confidence intervals associated with the gross and net technical efficiency measures are generally sufficiently wide to prohibit too strict a reliance on the accuracy of the point estimates.

\section{CONCLUSIONS.}

In using DEA techniques for the analysis of efficiency a distinction can be drawn between gross and net technical efficiency. The latter concept corresponds to a non-radial measure of efficiency that takes separate of account of slack in the use of employed resources. In the approach outlined in this article, a measure of net technical efficiency is the product

\footnotetext{
${ }^{3}$ Another possible source of inefficiency not considered here is allocative inefficiency.
} 
of a slack efficiency measure and a standard input oriented (gross) technical efficiency measure. In order to assess the reliability of these measures, some assessment of their statistical properties is required. In the current state of knowledge, such an assessment is only possible using simulation methods such as the bootstrap. Implementation of the bootstrap in this context requires some care, as has been shown by Wilson and Simar (1995). This article has shown how to extend bootstrap methods developed for gross technical efficiency measurement to measures of slack and net technical efficiency. The practicality of the method was exemplified using the Christensen and Greene (1976) data set on electricity production in the US. The results indicated that slack efficiency estimates are quite high, typically of the order of $90 \%$ or above, compared with gross and net technical efficiencies that averaged around $80 \%$ and $76 \%$ respectively in the sample. Analysis of the bootstrap simulation suggests that, at least for this data set, technical efficiency measures have a small downward bias and that the confidence intervals obtainable from the bootstrap analysis, although wide enough to suggest cautious interpretation of the efficiency measures, are nonetheless good indicators of the likely order of magnitude of the efficiency measures. These results enhance the usefulness of the DEA approach to efficiency measurement by demonstrating the viability of bootstrap methods in performing statistical assessments of the resulting efficiency measures. 
Table 1.

Gross Technical Efficiency: results for subsample.

\begin{tabular}{|c|c|c|c|c|c|c|c|}
\hline$k$ & $\hat{\theta}_{k}$ & Bias & $\tilde{\theta}_{k}$ & $R M S E$ & \multicolumn{2}{|c|}{$\begin{array}{c}\text { Confidence } \\
\text { Interval }\end{array}$} & Rank \\
\hline 6 & 0.697 & 0.033 & 0.663 & 0.052 & 0.585 & 0.741 & 123 \\
\hline 37 & 0.775 & 0.031 & 0.744 & 0.050 & 0.666 & 0.822 & 96 \\
\hline 48 & 0.846 & 0.026 & 0.820 & 0.047 & 0.746 & 0.894 & 55 \\
\hline 53 & 0.801 & 0.030 & 0.771 & 0.052 & 0.690 & 0.852 & 76 \\
\hline 54 & 0.837 & 0.029 & 0.807 & 0.051 & 0.726 & 0.888 & 60 \\
\hline 58 & 0.962 & 0.015 & 0.947 & 0.031 & 0.892 & 1.000 & 19 \\
\hline 71 & 0.896 & 0.027 & 0.869 & 0.047 & 0.792 & 0.947 & 33 \\
\hline 72 & 0.744 & 0.034 & 0.710 & 0.054 & 0.631 & 0.789 & 111 \\
\hline 85 & 0.597 & 0.032 & 0.565 & 0.051 & 0.490 & 0.640 & 146 \\
\hline 100 & 0.763 & 0.034 & 0.729 & 0.053 & 0.648 & 0.811 & 101 \\
\hline 113 & 0.874 & 0.030 & 0.845 & 0.052 & 0.763 & 0.927 & 43 \\
\hline 119 & 0.802 & 0.032 & 0.770 & 0.052 & 0.690 & 0.851 & 75 \\
\hline 120 & 0.883 & 0.028 & 0.854 & 0.050 & 0.774 & 0.935 & 39 \\
\hline 149 & 0.806 & 0.029 & 0.777 & 0.050 & 0.697 & 0.857 & 71 \\
\hline 155 & 0.783 & 0.031 & 0.752 & 0.054 & 0.667 & 0.837 & 91 \\
\hline
\end{tabular}


Table 2

Slack Efficiency: results for subsample.

\begin{tabular}{|c|c|c|c|c|c|c|c|}
\hline$k$ & $S E_{k}$ & Bias & $\begin{array}{r}S E_{k} \\
(\mathrm{BC})\end{array}$ & RMSE & \multicolumn{2}{|c|}{$\begin{array}{c}\text { Confidence } \\
\text { Interval }\end{array}$} & Rank \\
\hline 6 & 0.896 & 0.010 & 0.886 & 0.019 & 0.855 & 0.917 & 133 \\
\hline 37 & 1.000 & 0.000 & 1.000 & 0.004 & 0.996 & 1.000 & 38 \\
\hline 48 & 1.000 & 0.000 & 1.000 & 0.000 & 1.000 & 1.000 & 33 \\
\hline 53 & 1.000 & 0.000 & 1.000 & 0.000 & 1.000 & 1.000 & 52 \\
\hline 54 & 0.964 & 0.011 & 0.953 & 0.018 & 0.928 & 0.978 & 105 \\
\hline 58 & 1.000 & 0.000 & 1.000 & 0.003 & 0.996 & 1.000 & 29 \\
\hline 71 & 1.000 & 0.000 & 1.000 & 0.001 & 1.000 & 1.000 & 24 \\
\hline 72 & 1.000 & 0.000 & 1.000 & 0.015 & 0.958 & 1.000 & 51 \\
\hline 85 & 0.853 & 0.014 & 0.839 & 0.026 & 0.796 & 0.883 & 143 \\
\hline 100 & 0.945 & 0.014 & 0.931 & 0.019 & 0.908 & 0.954 & 116 \\
\hline 113 & 0.969 & 0.000 & 0.969 & 0.013 & 0.946 & 0.992 & 102 \\
\hline 119 & 1.000 & 0.000 & 1.000 & 0.000 & 1.000 & 1.000 & 58 \\
\hline 120 & 1.000 & 0.000 & 1.000 & 0.011 & 0.973 & 1.000 & 14 \\
\hline 149 & 1.000 & 0.000 & 1.000 & 0.000 & 1.000 & 1.000 & 3 \\
\hline 155 & 0.961 & 0.024 & 0.937 & 0.028 & 0.909 & 0.965 & 106 \\
\hline
\end{tabular}


Table 3

Net Technical Efficiency: results for subsample.

\begin{tabular}{|c|c|c|c|c|c|c|c|}
\hline$k$ & $T E_{k}^{N}$ & Bias & $\begin{array}{l}T E_{k}^{N} \\
(\mathrm{BC})\end{array}$ & RMSE & \multicolumn{2}{|c|}{$\begin{array}{c}\text { Confidence } \\
\text { Interval }\end{array}$} & Rank \\
\hline 6 & 0.624 & 0.037 & 0.587 & 0.055 & 0.507 & 0.667 & 129 \\
\hline 37 & 0.775 & 0.031 & 0.744 & 0.050 & 0.665 & 0.824 & 84 \\
\hline 48 & 0.846 & 0.026 & 0.820 & 0.047 & 0.746 & 0.894 & 46 \\
\hline 53 & 0.801 & 0.030 & 0.771 & 0.052 & 0.690 & 0.852 & 68 \\
\hline 54 & 0.807 & 0.038 & 0.769 & 0.056 & 0.689 & 0.848 & 62 \\
\hline 58 & 0.962 & 0.015 & 0.947 & 0.031 & 0.891 & 1.000 & 16 \\
\hline 71 & 0.896 & 0.026 & 0.869 & 0.047 & 0.792 & 0.947 & 28 \\
\hline 72 & 0.744 & 0.024 & 0.719 & 0.050 & 0.634 & 0.805 & 99 \\
\hline 85 & 0.509 & 0.036 & 0.473 & 0.053 & 0.399 & 0.547 & 145 \\
\hline 100 & 0.721 & 0.043 & 0.678 & 0.060 & 0.597 & 0.759 & 106 \\
\hline 113 & 0.847 & 0.028 & 0.819 & 0.052 & 0.732 & 0.907 & 45 \\
\hline 119 & 0.802 & 0.032 & 0.770 & 0.052 & 0.690 & 0.851 & 66 \\
\hline 120 & 0.883 & 0.022 & 0.861 & 0.047 & 0.780 & 0.942 & 33 \\
\hline 149 & 0.806 & 0.029 & 0.777 & 0.050 & 0.697 & 0.857 & 63 \\
\hline 155 & 0.752 & 0.049 & 0.703 & 0.068 & 0.614 & 0.791 & 95 \\
\hline
\end{tabular}


Table 4.

Averages over Full Sample.

\begin{tabular}{l|ccccc}
\hline $\begin{array}{l}\text { Efficiency } \\
\text { Index }\end{array}$ & Estimate & Bias & $\begin{array}{c}\text { Estimate } \\
(\mathrm{BC})\end{array}$ & $R M S E$ & $\begin{array}{c}\text { Width } \\
\mathrm{CI}\end{array}$ \\
\hline$T E$ & 0.798 & 0.027 & 0.772 & 0.046 & 0.143 \\
$S E$ & 0.956 & 0.014 & 0.942 & 0.023 & 0.060 \\
$T E^{N}$ & 0.765 & 0.036 & 0.729 & 0.057 & 0.164 \\
\hline
\end{tabular}




\section{APPENDIX.}

Assume that $n$ observations $v_{1}, \ldots, v_{n}$ of a continuous random variable $V$ are available. Consider a consistent kernel density estimator of the density function $f$ of $V$ given by

$$
\hat{f}(v)=\frac{1}{n b} \sum_{i=1}^{n} K\left(\frac{v-v_{i}}{b}\right)
$$

where $K$ is a continuous and symmetric density function with mean zero and variance equal to unity and $b$ is a bandwidth parameter. Let

$$
V_{j}^{*}=v_{I_{j}}+b \epsilon_{j}
$$

where the $I_{j}$ are independent and uniformly distributed on the integers $1, \ldots, n$ and the $\epsilon_{j}$ are a random sample from a variable with density $K$ and are independent of the $I_{j}$. It is easy to show that the density function for $V_{j}^{*}$ is (see (25))

$$
f_{V_{j}^{*}}(v)=\hat{f}(v)
$$

In the case in which the support of $V$ is bounded below by zero, let $T_{j}=\left|V_{j}^{*}\right|$. The density function for $T_{j}$ is

$$
\begin{aligned}
f_{T_{j}}(t) & =f_{V_{j}^{*}}(t)+f_{V_{j}^{*}}(-t) \\
& =\hat{f}(t)+\hat{f}(-t) \quad t \geq 0 \\
& =0 \quad \text { otherwise. }
\end{aligned}
$$

Since $\hat{f}(v)$ is a consistent estimator of $f(v)$, and since $f(v)=0$ for $v<0$, then, as the

sample size $n$ becomes large, $\hat{f}(-t)$ converges in probabilitv to zero when $t>0$. Thus $f_{T_{j}}(t)$ converges in probability to $f(t)$; in other words the density function for $T_{j}$ is a consistent estimator of the density function for $V$. This argument applies to (27) with $\hat{\gamma}_{i}^{+}$corresponding to $T$ and $\hat{\gamma}_{i}$ corresponding to $V$.

A similar argument applies to (29). In this case assume that $V$ is bounded above by 1 and now define

$$
\begin{aligned}
S_{j} & =V_{j}^{*} \quad \text { if } V_{j}^{*} \leq 1 \\
& =2-V_{j}^{*} \quad \text { if } V_{j}^{*}>1 .
\end{aligned}
$$


Then it is straightforward to show that the density function for $S_{j}$ is

$$
\begin{aligned}
f_{S_{j}}(s) & =f_{V_{j}^{*}}(s)+f_{V_{j}^{*}}(2-s) \\
& =\hat{f}(s)+\hat{f}(2-s), \quad s \leq 1 \\
& =0, \quad s>1 .
\end{aligned}
$$

Since by assumption in this case $V \leq 1$ then $f(v)=0$ if $v>1$. Thus if $s \leq 1$ then $2-s>1$ and $f(2-s)=0$. Hence, since $\hat{f}(v)$ is a consistent estimator of $f(v)$, it follows that $f_{S_{j}}(s)$ converges in probability to $f(s)$. Apply this argument to $(29)$ with $\hat{\theta}^{+}$ corresponding to $S$ and $\hat{\theta}$ to $V$. 


\section{References}

BANkeR, R. D. (1993). Maximum likelihood, consistency and data envelopment analysis: A statistical foundation. Management Science, 39, 1265-1273.

Charnes, A., Cooper, W., Lewin, A. Y. and Seiford, L. M. (eds.) (1997). Data Envelopment Analysis: Theory, Methodology, and Application. Kluwer Academic Publishers.

Christensen, L. and Greene, W. H. (1976). Economies of scale in U. S. electric power generation. Journal of Political Economy, 84, 655-676.

Davison, A. C. and Hinkley, D. V. (1997). Bootstrap Methods and their Application. Cambridge University Press, Cambridge, U. K.

Debreu, G. (1951). The coefficient of resource utilization. Econometrica, 19, 273-292.

Efron, B. and Tibshirani, R. J. (1993). An Introduction to the Bootstrap. Chapman and Hall, London.

Färe, R., Grosskopf, S. and Lovell, C. A. K. (1994). Production Frontiers. Cambridge University Press, Cambridge, U. K.

FARrell, M. J. (1957). The measurement of productive efficiency. Journal of the Royal Statistical Society, Series A, 120, 253-281.

Gijbels, I., Mammen, E., Park, B. U. and Simar, L. (1999). On estimation of monotone and concave frontier functions. Journal of the American Statistical Association, 94, 220-228.

Grimmett, G. and Stirzaker, D. R. (1992). Probability and Random Processes. Oxford University Press, Oxford, 2nd edition.

Grosskopf, S. (1996). Statistical inference and nonparametric efficiency: A selective survey. Journal of Productivity Analysis, 7, 161-176. 
Hall, P. (1992). The Bootstrap and Edgeworth Expansion. Springer-Verlag, New York.

Kneip, A., Park, B. U. and Simar, L. (1998). A note on the convergence of nonparametric dea estimators for production efficiency scores. Econometric Theory, 14, $783-793$.

Koopmans, T. C. (1951). An analysis of production as an efficient combination of activities. In T. C. Koopmans (ed.), Activity Analysis of Production and Allocation, Cowles Commission for Research in Economics, Monograph No. 13. Wiley, New York.

Lynde, C. and Richmond, J. (2000). Measuring efficiency and costing slack. Department of Economics, University of Essex, Discussion Paper No. 514.

Silverman, B. W. (1986). Density Estimation in Statistics and Data Analysis. Chapman and Hall, London.

SimAR, L. (1996). Aspects of statistical analysis in DEA-type frontier models. Journal of Productivity Analysis, 7, 177-185.

Simar, L. and Wilson, P. W. (1998). Sensitivity analysis of efficiency scores: How to bootstrap in nonparametric frontier models. Management Science, 44, 49-61.

Simar, L. and Wilson, P. W. (1999). Statistical inference in nonparametric frontier models: The state of the art. Journal of Productivity Analysis, 11, 67-80.

SimAR, L. and Wilson, P. W. (2000). A general methodology for bootstrapping in non-parametric frontier models. Journal of Applied Statistics, 27, 779-802.

Wilson, P. W. and Simar, L. (1995). Bootstrap estimation for nonparametric efficiency estimates. CORE Discussion Paper, No. 9571. 